Proyecciones Journal of Mathematics

Vol. 33, No 1, pp. 77-90, March 2014.

Universidad Católica del Norte

Antofagasta - Chile

\title{
Some Umbral Calculus Presentations of the Chan-Chyan-Srivastava Polynomials and the Erkuş-Srivastava Polynomials
}

\author{
H. M. Srivastava \\ University of Victoria, Canada \\ K. S. Nisar \\ Salman Bin Abdu-Aziz University, Saudi Arabia \\ and \\ Mumtaz Ahmad Khan \\ Aligarh Muslim University, India \\ Received : February 2013. Accepted : March 2013
}

\begin{abstract}
In their recent investigation involving differential operators for the generalized Lagrange polynomials, Chan et. al. [3] encountered and proved a certain summation identity and several other results for the Lagrange polynomials in several variables, which are popularly known in the literature as the Chan-Chyan-Srivastava polynomials. These multivariable polynomials have been studied systematically and extensively in the literature ever since then (see, for example, [1], [4], [9], [11], [12] and [13]). In the present paper, we investigate umbral calculus presentations of the Chan-Chyan-Srivastava polynomials and also of their substantially more general form, the Erkus-Srivastava polynomials [9]. Some other closely-related results are also considered.
\end{abstract}

2010 Mathematics Subject Classification : Primary 33C45, 33C65; Secondary $33 C 05$.

Key Words and Phrases : Lagrange polynomials; Hermite-Kampé de Fériet polynomials; Lagrange-Hermite polynomials; Chan-ChyanSrivastava polynomials; Erkus-Srivastava polynomials; Umbral calculus; Pochhammer symbol; Multinomial theorem and multinomial coefficients; Principle of monoumbrality; Monoumbral expansions. 


\section{Introduction, Definitions and Notations}

The familiar (two-variable) polynomials $g_{n}^{(\alpha, \beta)}(x, y)$, which are generated by

$$
\begin{gathered}
(1-x z)^{-\alpha}(1-y z)^{-\beta}=\sum_{n=0}^{\infty} g_{n}^{(\alpha, \beta)}(x, y) z^{n} \\
\left(\alpha, \beta \in \mathbf{C} ;|z|<\min \left\{|x|^{-1},|y|^{-1}\right\}\right),
\end{gathered}
$$

are known as the Lagrange polynomials which occur in certain problems in statistics (cf., e.g., Erdélyi et al. [8, p. 267]; see also [15, p. 441 et seq.]).

The (three-variable) Lagrange polynomials $g_{n}^{(\alpha, \beta, \gamma)}(x, y, z)$, which are defined by means of the generating function:

$$
\begin{gathered}
(1-x t)^{-\alpha}(1-y t)^{-\beta}(1-z t)^{-\gamma}=\sum_{n=0}^{\infty} g_{n}^{(\alpha, \beta, \gamma)}(x, y, z) \frac{t^{n}}{n !} \\
\left(\alpha, \beta, \gamma \in \mathbf{C} ;|t|<\min \left\{|x|^{-1},|y|^{-1},|z|^{-1}\right\}\right),
\end{gathered}
$$

were studied recently by Khan and Shukla [10]. Subsequently, Chan et al. [3] introduced and investigated the multivariable extension of the classical Lagrange polynomials $g_{n}^{(\alpha, \beta)}(x, y)$ generated by (1.1).

These multivariable Lagrange polynomials $g_{n}^{\left(\alpha_{1}, \cdots, \alpha_{r}\right)}\left(x_{1}, \cdots, x_{r}\right)$, which are popularly known in the literature as the Chan-Chyan-Srivastava polynomials, are generated by (see, for details, [3]; see also [4], [12] and [13])

$$
\begin{gathered}
\prod_{j=1}^{r}\left\{\left(1-x_{j} z\right)^{-\alpha_{j}}\right\}=\sum_{r=0}^{\infty} g_{n}^{\left(\alpha_{1}, \cdots, \alpha_{r}\right)}\left(x_{1}, \cdots, x_{r}\right) z^{n} \\
\left(\alpha_{j} \in \mathbf{C} \quad(j=1, \cdots, r) ;|z|<\min \left\{\left|x_{1}\right|^{-1}, \cdots,\left|x_{r}\right|^{-1}\right\}\right),
\end{gathered}
$$

so that, upon comparison with the generating function (1.2), we have the following relationship:

$$
\begin{gathered}
g_{n}^{(\alpha, \beta, \gamma)}(x, y, z)=n ! g_{n}^{(\alpha, \beta, \gamma)}(x, y, z) \\
\left(n \in \mathbf{N}_{0}:=\mathbf{N} \cup\{0\} ; \mathbf{N}:=\{1,2,3, \cdots\}\right)
\end{gathered}
$$

with the (three-variable) Lagrange polynomials $g_{n}^{(\alpha, \beta, \gamma)}(x, y, z)$ studied by Khan and Shukla [10].

Clearly, the defining generating function (1.3) yields the explicit representation given by [3, p. 140, Eq. (6)] 


$$
\begin{gathered}
g_{n}^{\left(\alpha_{1}, \cdots, \alpha_{r}\right)}\left(x_{1}, \cdots, x_{r}\right)=\sum_{\begin{array}{c}
k_{1}, \cdots, k_{r} \in \mathbf{N}_{0} \\
\left(k_{1}+\cdots+k_{r}=n\right)
\end{array}}\left(\alpha_{1}\right)_{k_{1}} \cdots\left(\alpha_{r}\right)_{k_{r}} \frac{x_{1}^{k_{1}}}{k_{1} !} \cdots \frac{x_{r}^{k_{r}}}{k_{r} !} \\
=\sum_{k_{1}, \cdots, k_{r-1}=0}^{k_{1}+\cdots+k_{r-1}<n}=\frac{\left(\alpha_{1}\right)_{n-k_{1}-\cdots-k_{r-1}}\left(\alpha_{2}\right)_{k_{1}} \cdots\left(\alpha_{r}\right)_{k_{r-1}}}{\left(n-k_{1}-\cdots-k_{r}-1\right) ! k_{1} ! \cdots k_{r-1} !} \\
(1.4) \quad x_{1}^{n-k_{1}-\cdots-k_{r-1}} x_{2}^{k_{1}} \cdots x_{r}^{k_{r-1}}
\end{gathered}
$$

or, equivalently, by $[11$, p. 522 , Eq. (17)]

$$
\begin{gathered}
g_{n}^{\left(\alpha_{1}, \cdots, \alpha_{r}\right)}\left(x_{1}, \cdots, x_{r}\right)=\sum_{n_{r-1}=0}^{n} \sum_{n_{r-2}=0}^{n_{r-1}} \cdots \sum_{n_{1}=0}^{n_{2}} \frac{\left(\alpha_{1}\right)_{n_{1}}\left(\alpha_{2}\right)_{n_{2}-n_{1} \cdots\left(\alpha_{r}\right)_{n-n_{r-1}}}}{n_{1} !\left(n_{2}-n_{1}\right) ! \cdots\left(n-n_{r-1}\right) !} \\
=\sum_{k_{1}=0}^{n} \sum_{k_{2}=0}^{k_{1}} \ldots \sum_{k_{r-1}=0}^{n_{1}} x_{2}^{n_{2}-n_{1}} \cdots x_{r}^{n-n_{r-1}} \\
\text { 5) } \\
\cdot x_{1}^{n-k_{1}} x_{2}^{k_{2}-k_{1}} \cdots x_{r}^{k_{r-1}}
\end{gathered}
$$

where, as usual, $(\lambda)_{n}$ denotes the Pochhammer symbol given by

$$
(\lambda)_{0}:=1 \quad \text { and } \quad(\lambda)_{n}:=\lambda(\lambda+1) \cdots(\lambda+n-1) \quad(n \in \mathbf{N}) .
$$

Altın and Erkus [1] presented a multivariable extension of the socalled Lagrange-Hermite polynomials generated by (see [1, p. 239, Eq. $(1.2)])$

$$
\begin{gathered}
\prod_{j=1}^{r}\left\{\left(1-x_{j} z^{j}\right)^{-\alpha_{j}}\right\}=\sum_{n=0}^{\infty} h_{n}^{\left(\alpha_{1}, \cdots, \alpha_{r}\right)}\left(x_{1}, \cdots, x_{r}\right) z^{n} \\
\left(\alpha_{j} \in \mathbf{C} \quad(j=1, \cdots, r) ;|z|<\min _{j \in\{1, \cdots, r\}}\left\{\left|x_{j}\right|^{-1 / j}\right\}\right) .
\end{gathered}
$$

The case $r=2$ of the polynomials given by (1.6) corresponds to the familiar (two-variable) Lagrange-Hermite polynomials considered by Dattoli et al. [6]. 
The multivariable (Erkuş-Srivastava) polynomials $\mathcal{U}_{n ; \ell_{1}, \cdots, \ell_{r}}^{\left(\alpha_{1}, \cdots, \alpha_{r}\right)}\left(x_{1}, \cdots, x_{r}\right)$, which are defined by the following generating function [9, p. 268, Eq. (3)]:

$$
\prod_{j=1}^{r}\left\{\left(1-x_{j} z^{\ell_{j}}\right)^{-\alpha_{j}}\right\}=\sum_{n=0}^{\infty} \mathcal{U}_{n ; \ell_{1}, \cdots, \ell_{r}}^{\left(\alpha_{1}, \cdots, \alpha_{r}\right)}\left(x_{1}, \cdots, x_{r}\right) z^{n}
$$$$
\left(\alpha_{j} \in \mathbf{C} \quad(j=1, \cdots, r) ; \ell_{j} \in \mathbf{N} \quad(j=1, \cdots, r) ;\right.
$$

$$
\left.|z|<\min \left\{\left|x_{1}\right|^{-1 / \ell_{1}}, \cdots,\left|x_{r}\right|^{-1 / \ell_{r}}\right\}\right) \text {, }
$$

are a unification (and generalization) of several known families of multivariable polynomials including (for example) the Chan-Chyan-Srivastava polynomials $g_{n}^{\left(\alpha_{1}, \cdots, \alpha_{r}\right)}\left(x_{1}, \cdots, x_{r}\right)$ defined by (1.3) (see, for details, [9]). Obviously, the Chan-Chyan-Srivastava polynomials $g_{n}^{\left(\alpha_{1}, \cdots, \alpha_{r}\right)}\left(x_{1}, \cdots, x_{r}\right)$ follow as the special case of the Erkuş-Srivastava polynomials $\mathcal{U}_{n ; \ell_{1}, \cdots, \ell_{r}}^{\left(\alpha_{1}, \cdots, \alpha_{r}\right)}\left(x_{1}, \cdots, x_{r}\right)$ when $\ell_{j}=1(j=1, \cdots, r)$.

Moreover, the Lagrange-Hermite polynomials $h_{n}^{\left(\alpha_{1}, \cdots, \alpha_{r}\right)}\left(x_{1}, \cdots, x_{r}\right)$ follow as a special case of the Erkus-Srivastava polynomials $\mathcal{U}_{n ; \ell_{1}, \cdots, \ell_{r}}^{\left(\alpha_{1}, \cdots, \alpha_{r}\right)}\left(x_{1}, \cdots, x_{r}\right)$ when $\ell_{j}=j \quad(j=1, \cdots, r)$.

The generating function (1.7) yields the following explicit representation [9, p. 268, Eq. (4)]:

$$
\mathcal{U}_{n ; \ell_{1}, \cdots, \ell_{r}}^{\left(\alpha_{1}, \cdots, \alpha_{r}\right)}\left(x_{1}, \cdots, x_{r}\right)=\sum_{\substack{k_{1}, \cdots, k_{r} \in \mathbf{N}_{0} \\\left(\ell_{1} k_{1}+\cdots+\ell_{r} k_{r}=n\right)}}\left(\alpha_{1}\right)_{k_{1}} \cdots\left(\alpha_{r}\right)_{k_{r}} \frac{x_{1}^{k_{1}}}{k_{1} !} \cdots \frac{x_{r}^{k_{r}}}{k_{r} !},
$$

which, in the special case when $\ell_{j}=1(j=1, \cdots, r)$, corresponds to the first expression in (1.4).

Each of the above families of multivariable polynomials has been investigated systematically and extensively in the literature ever since the publication of the work by Chan et al. [9] (see, for example, [1], [4], [9], [11], [12] and [13]). The main objective of the present paper is to derive umbral calculus presentations of the Chan-Chyan-Srivastava polynomials $g_{n}^{\left(\alpha_{1}, \cdots, \alpha_{r}\right)}\left(x_{1}, \cdots, x_{r}\right)$ generated by (1.3) and also of the substantially more general Erkuş-Srivastava polynomials $\mathcal{U}_{n ; \ell_{1}, \cdots, \ell_{r}}^{\left(\alpha_{1}, \cdots, \alpha_{r}\right)}\left(x_{1}, \cdots, x_{r}\right)$ generated by (1.7). Upon suitable specialization, this last umbral calculus presentation is shown to yield the corresponding result for the polynomials $h_{n}^{\left(\alpha_{1}, \cdots, \alpha_{r}\right)}\left(x_{1}, \cdots, x_{r}\right)$ generated by (1.6). 


\section{Umbral Calculus Presentations}

The Chan-Chyan-Srivastava polynomials in (1.3) exhibits a structure which, according to the prescription provided in [7], can be viewed as the umbral image of ordinary monomials. Indeed, by using the generating function in (1.3) and an elementary integral identity in the form [2] :

$$
\kappa^{-\nu}=\frac{1}{\Gamma(\nu)} \int_{0}^{\infty} e^{-\kappa t} t^{\nu-1} d t \quad(\min \{\Re(\kappa), \Re(\nu)\}>0),
$$

we can easily derive the following integral representation of the ChanChyan-Srivastava polynomials:

$$
\begin{gathered}
g_{n}^{\left(\alpha_{1}, \cdots, \alpha_{r}\right)}\left(x_{1}, \cdots, x_{r}\right)=\frac{1}{\Gamma\left(\alpha_{1}\right) \cdots \Gamma\left(\alpha_{r}\right)} \int_{0}^{\infty} \cdots \int_{0}^{\infty} e^{-\left(\xi_{1}+\cdots+\xi_{r}\right)} \\
\cdot \xi_{1}^{\alpha_{1}-1} \cdots \xi_{r}^{\alpha_{r}-1} \mathcal{P}_{n}\left(x_{1} \xi_{1}, \cdots, x_{r} \xi_{r}\right) d \xi_{1} \cdots d \xi_{r}
\end{gathered}
$$

where

$$
\begin{aligned}
& \mathcal{P}_{n}\left(x_{1}, \cdots, x_{r}\right):=\frac{\left(x_{1}+\cdots+x_{r}\right)^{n}}{n !} \\
&=\frac{1}{n !} \sum_{\substack{k_{1}, \cdots, k_{r} \in \mathbf{N}_{0} \\
\left(k_{1}+\cdots+k_{r}=n\right)}}\left(\begin{array}{c}
n \\
k_{1}, \ldots, k_{r}
\end{array}\right) x_{1}^{k_{1}} \cdots x_{r}^{k_{r}}
\end{aligned}
$$

in terms of the multinomial coefficients given by

$$
\left(\begin{array}{c}
n \\
k_{1}, \cdots, k_{r}
\end{array}\right):=\frac{n !}{k_{1} ! \cdots k_{r} !} \quad\left(n, k_{1}, \cdots, k_{r} \in \mathbf{N}_{0}\right) .
$$

Alternatively, the multinomial theorem (see, for example, [15, p. 87, Problem 5]) used in (2.3) can indeed be restated as follows:

$$
\begin{gathered}
\mathcal{P}_{n}\left(x_{1}, \cdots, x_{r}\right)=\frac{1}{n !} \sum_{k_{1}=0}^{n} \sum_{k_{2}=0}^{n-k_{1}} \ldots \sum_{k_{r-1}=0}^{n-k_{1}-\cdots-k_{r-2}}\left(\begin{array}{c}
n \\
k_{1}
\end{array}\right)\left(\begin{array}{c}
n-k_{1} \\
k_{2}
\end{array}\right) \\
\cdots\left(\begin{array}{c}
n-k_{1}-\cdots-k_{r-2} \\
k_{r-1}
\end{array}\right) \\
\cdot x_{1}^{n-k_{1}-\cdots-k_{r-1}} x_{2}^{k_{1}} \cdots x_{r}^{k_{r-1}} .
\end{gathered}
$$


It is immediately seen from the integral representation (2.2) that [see Eq. (1.4)]

$$
\begin{aligned}
g_{n}^{\left(\alpha_{1}, \cdots, \alpha_{r}\right)}\left(x_{1}, \cdots, x_{r}\right)= & \sum_{\substack{k_{1}, \cdots, k_{r} \in \mathbf{N}_{0} \\
\left(k_{1}+\cdots+k_{r}=n\right)}}\left(\begin{array}{c}
\alpha_{1}+k_{1}-1 \\
k_{1}
\end{array}\right) \cdots\left(\begin{array}{c}
\alpha_{r}+k_{r}-1 \\
k_{r}
\end{array}\right) x_{1}^{k_{1}} \ldots x_{r}^{k_{r}} \\
= & \sum_{k_{1}=0}^{n} \sum_{k_{2}=0}^{n-k_{1}} \cdots \sum_{k_{r-1}=0}^{n-k_{1}-\cdots-k_{r-2}} \frac{\left(\alpha_{1}\right)_{n-k_{1}-\cdots-k_{r-1}}\left(\alpha_{2}\right)_{k_{1}} \cdots\left(\alpha_{r}\right)_{k_{r-1}}}{\left(n-k_{1}-\cdots-k_{r-1}\right) ! k_{1} ! \cdots k_{r-2} !} \\
(2.5) & \cdot x_{1}^{n-k_{1}-\cdots-k_{r-1}} x_{2}^{k_{1}} \cdots x_{r}^{k_{r-1}},
\end{aligned}
$$

which follows also from the second explicit expression in (1.4).

We now define the umbral quantities $\left\langle\alpha_{1} x_{1}\right\rangle, \cdots,\left\langle\alpha_{r} x_{r}\right\rangle$ together with their properties given by (see, for details, [5] ; see also [14])

$$
\left\langle\alpha_{j} x_{j}\right\rangle^{n}=\left(\alpha_{j}\right)_{n} x_{j}^{n} \quad\left(j=1, \cdots, r ; n \in \mathbf{N}_{0}\right)
$$

and the pairs of operators $\hat{X}_{j}$ and $\hat{P}_{x_{j}}(j=1, \cdots, r)$ such that

$$
\begin{gathered}
\hat{X}_{j}\left\{\left\langle\alpha_{j} x_{j}\right\rangle^{n}\right\}=\left\langle\alpha_{j} x_{j}\right\rangle^{n+1} \quad \text { and } \quad \hat{P}_{x_{j}}\left\{\left\langle\alpha_{j} x_{j}\right\rangle^{n}\right\}=n\left\langle\alpha_{j} x_{j}\right\rangle^{n-1} \\
(j=1, \cdots, r),
\end{gathered}
$$

so that, obviously,

$$
\begin{gathered}
\hat{X}_{j} \hat{P}_{x_{j}}\left\{\left\langle\alpha_{j} x_{j}\right\rangle^{n}\right\}=n\left\langle\alpha_{j} x_{j}\right\rangle^{n} \quad \text { and } \quad \hat{P}_{x_{j}} \hat{X}_{j}\left\{\left\langle\alpha_{j} x_{j}\right\rangle^{n}\right\}=(n+1)\left\langle\alpha_{j} x_{j}\right\rangle^{n} \\
(j=1, \cdots, r) .
\end{gathered}
$$

We thus find that the Chan-Chyan-Srivastava polynomials $g_{n}^{\left(\alpha_{1}, \cdots, \alpha_{r}\right)}\left(x_{1}, \cdots, x_{r}\right)$ satisfy the following operational formula:

$$
\begin{gathered}
\left(\hat{X}_{1} \hat{P}_{x_{1}}+\cdots+\hat{X}_{r} \hat{P}_{x_{r}}\right)\left\{g_{n}^{\left(\alpha_{1}, \cdots, \alpha_{r}\right)}\left(x_{1}, \cdots, x_{r}\right)\right\} \\
=n g_{n}^{\left(\alpha_{1}, \cdots, \alpha_{r}\right)}\left(x_{1}, \cdots, x_{r}\right)
\end{gathered}
$$


which, in the special case when

$$
r=2, \quad \alpha_{1}=\alpha, \quad \alpha_{2}=\beta, \quad x_{1}=x \quad \text { and } \quad x_{2}=y,
$$

would provide the corrected version of a result stated by Dattoli et al. [6, p. 182, Eq. (10)].

It is also clear that the umbral image of the generating function (1.3) is given by

$$
e^{\left[\left\langle\alpha_{1} x_{1}\right\rangle+\cdots+\left\langle\alpha_{r} x_{r}\right\rangle\right] z}=\sum_{n=0}^{\infty} g_{n}^{\left(\alpha_{1}, \cdots, \alpha_{r}\right)}\left(x_{1}, \cdots, x_{r}\right) z^{n} .
$$

In our next section (Section 3), we will show that the above notion and formalism of monoumbrality (that is, monomiality together with umbrality) can be extended to a large family of polynomials as well as of functions and that this principle of monoumbrality will provide a powerful tool for simplifying calculations (see also [5], [6] and [7]).

\section{Applications of the Principle of Monoumbrality}

In the preceding section, we have noted that the umbral image of the generating function (1.3) of the Chan-Chyan-Srivastava polynomials

$g_{n}^{\left(\alpha_{1}, \cdots, \alpha_{r}\right)}\left(x_{1}, \cdots, x_{r}\right)$ is, in fact, of the exponential type given by $(2.7)$. Here, in this section, we begin by recalling the following easily understandable decomposition rules for the umbral algebra (see [5], [6] and [7]):

$$
e^{\left\langle\alpha_{1} x_{1}\right\rangle+\cdots+\left\langle\alpha_{r} x_{r}\right\rangle}=e^{\left\langle\alpha_{1} x_{1}\right\rangle} \cdots e^{\left\langle\alpha_{r} x_{r}\right\rangle},
$$

$$
e^{\left[\left\langle\alpha\left(x_{1}+\cdots+x_{r}\right)\right\rangle\right]} \neq e^{\left\langle\alpha x_{1}\right\rangle} \cdots e^{\left\langle\alpha x_{r}\right\rangle},
$$

that is, more explicitly,

$$
e^{\left[\left\langle\alpha\left(x_{1}+\cdots+x_{r}\right)\right\rangle\right]} \neq\left(\sum_{k_{1}=0}^{\infty}(\alpha)_{k_{1}} \frac{x_{1}^{k_{1}}}{k_{1} !}\right) \cdots\left(\sum_{k_{r}=0}^{\infty}(\alpha)_{k_{r}} \frac{x_{r}^{k_{r}}}{k_{r} !}\right) .
$$

We also note that

$$
\begin{gathered}
e^{\left\langle\alpha_{1} x_{1}\right\rangle z}=e^{\left\langle\alpha_{1} x_{1}\right\rangle z+\cdots+\left\langle\alpha_{r} x_{r}\right\rangle z} \cdot e^{\left\langle-\alpha_{2} x_{2}\right\rangle z} \cdots e^{\left\langle-\alpha_{r} x_{r}\right\rangle z} \\
=e^{\left[\left\langle\alpha_{1} x_{1}\right\rangle+\cdots+\left\langle\alpha_{r} x_{r}\right\rangle\right] z} \cdot e^{\left[\left\langle-\alpha_{2} x_{2}\right\rangle+\cdots+\left\langle-\alpha_{r} x_{r}\right\rangle\right] z} .
\end{gathered}
$$


By applying this last umbral relationship (3.4) in conjunction with (2.5) and (2.7), we find that

$$
\begin{gathered}
\sum_{n=0}^{\infty}\left(\alpha_{1}\right)_{n} \frac{\left(x_{1} z\right)^{n}}{n !}=\left(\sum_{n=0}^{\infty} g_{n}^{\left(\alpha_{1}, \cdots, \alpha_{r}\right)}\left(x_{1}, \cdots, x_{r}\right) z^{n}\right) \\
\left(\sum_{n=0}^{\infty} g_{n}^{\left(-\alpha_{2}, \cdots,-\alpha_{r}\right)}\left(x_{2}, \cdots, x_{r}\right) z^{n}\right) \\
=\sum_{n=0}^{\infty} z^{n} \sum_{k=0}^{n} g_{k}^{\left(\alpha_{1}, \cdots, \alpha_{r}\right)}\left(x_{1}, \cdots, x_{r}\right) g_{n-k}^{\left(-\alpha_{2}, \cdots,-\alpha_{r}\right)}\left(x_{2}, \cdots, x_{r}\right),
\end{gathered}
$$

so that, upon equating the coefficients of $z^{n}$ from both sides in (3.5), we finally obtain

$$
\begin{gathered}
x_{1}^{n}=\frac{1}{\left(\alpha_{1}\right)_{n}} \sum_{k_{1}=0}^{n} \sum_{k_{2}=0}^{n-k_{1}} \cdots \sum_{k_{r-1}=0}^{n-k_{1}-\cdots-k_{r-2}} k_{1} !\left(\begin{array}{c}
n \\
k_{1}
\end{array}\right)\left(\begin{array}{c}
n-k_{1} \\
k_{2}
\end{array}\right) \\
\cdots\left(\begin{array}{c}
n-k_{1}-\cdots-k_{r-2} \\
k_{r-1}
\end{array}\right) \\
\cdot g_{k_{1}}^{\left(\alpha_{1}, \cdots, \alpha_{r}\right)}\left(x_{1}, \cdots, x_{r}\right)\left\langle-\alpha_{2} x_{2}\right\rangle^{n-k_{1}-\cdots-k_{r-1}}\left\langle-\alpha_{3} x_{3}\right\rangle^{k_{2}} \cdots\left\langle-\alpha_{r} x_{r}\right\rangle^{k_{r-1}},
\end{gathered}
$$

where, just as in the preceding section,

$$
\begin{gathered}
\left\langle-\alpha_{2} x_{2}\right\rangle^{n-k_{1}-\cdots-k_{r-1}}=\frac{\Gamma\left(-\alpha_{2}+n-k_{1}-\cdots-k_{r-1}\right)}{\Gamma\left(-\alpha_{2}\right)} x_{2}^{n-k_{1}-\cdots-k_{r-1}} \\
=\left(-\alpha_{2}\right)_{n-k_{1}-\cdots-k_{r-1}} x_{2}^{n-k_{1}-\cdots-k_{r-1}}
\end{gathered}
$$

and

$$
\left\langle-\alpha_{j} x_{j}\right\rangle^{k_{j-1}}=\frac{\Gamma\left(-\alpha_{j}+k_{j-1}\right)}{\Gamma\left(-\alpha_{j}\right)} x_{j}^{k_{j-1}}=\left(-\alpha_{j}\right)_{k_{j-1}} x_{j}^{k_{j-1}} \quad(j=3, \cdots, r) .
$$

Formula (3.6) provides the expansion of an ordinary monomial $x_{1}^{n}$ in terms of the Chan-Chyan-Srivastava polynomials

$$
g_{n}^{\left(\alpha_{1}, \cdots, \alpha_{r}\right)}\left(x_{1}, \cdots, x_{r}\right)
$$

involving such umbral quantities as those specified in (3.7) and (3.8). 
Next, for Erkuş-Srivastava polynomials $\mathcal{U}_{n ; \ell_{1}, \cdots, \ell_{r}}^{\left(\alpha_{1}, \cdots, \alpha_{r}\right)}\left(x_{1}, \cdots, x_{r}\right)$ generated by (1.7), it is easily seen that

$$
\begin{gathered}
\mathcal{U}_{n ; \ell_{1}, \cdots, \ell_{r}}^{\left(\alpha_{1}+\beta_{1}, \cdots, \alpha_{r}+\beta_{r}\right)}\left(x_{1}, \cdots, x_{r}\right) \\
=\sum_{k=0}^{n} \mathcal{U}_{n-k ; \ell_{1}, \cdots, \ell_{r}}^{\left(\alpha_{1}, \cdots, \alpha_{r}\right)}\left(x_{1}, \cdots, x_{r}\right) \mathcal{U}_{k ; \ell_{1}, \cdots, \ell_{r}}^{\left(\beta_{1}, \cdots, \beta_{r}\right)}\left(x_{1}, \cdots, x_{r}\right),
\end{gathered}
$$

which, for $\ell_{j}=1(j=1, \cdots, r)$, was given by Chan et al. [3, p. 147, Eq. (35)]. Moreover, the generating function (1.7) together with the integral formula (2.1) would yield the following analogue of the integral representation $(2.2)$ :

$$
\begin{gathered}
\mathcal{U}_{n ; \ell_{1}, \cdots, \ell_{r}}^{\left(\alpha_{1}+\beta_{1}, \cdots, \alpha_{r}+\beta_{r}\right)}\left(x_{1}, \cdots, x_{r}\right)=\frac{1}{\Gamma\left(\alpha_{1}\right) \cdots \Gamma\left(\alpha_{r}\right)} \int_{0}^{\infty} \cdots \int_{0}^{\infty} e^{-\left(\xi_{1}+\cdots+\xi_{r}\right)} \\
\cdot \xi_{1}^{\alpha_{1}-1} \cdots \xi_{r}^{\alpha_{r}-1} \mathcal{Q}_{n}\left(x_{1} \xi_{1}, \cdots, x_{r} \xi_{r}\right) d \xi_{1} \cdots d \xi_{r}
\end{gathered}
$$

where the polynomials $\mathcal{Q}_{n}\left(x_{1}, \cdots, x_{r}\right)$ are essentially the same as the multivariable Hermite-Kampé de Fériet polynomials given by

$$
\mathcal{Q}_{n}\left(x_{1}, \cdots, x_{r}\right):=\frac{1}{n !} \sum_{\substack{k_{1}, \cdots, k_{r} \in \mathbf{N}_{0} \\
\left(\ell_{1} k_{1}+\cdots+\ell_{r} k_{r}=n\right)}}\left(\begin{array}{c}
n \\
k_{1}, \cdots, k_{r}
\end{array}\right) x_{1}^{k_{1}} \cdots x_{r}^{k_{r}}
$$

in terms of the multinomial coefficients involved also in (2.3).

If we now make use of the principle of monoumbrality as detailed above, we can show similarly that

$$
\begin{gathered}
x_{1}^{n}=\frac{n !}{\left(\alpha_{1}\right)_{n}} \sum_{k_{1}=0}^{\ell_{1} n} \sum_{\begin{array}{c}
k_{2}, \cdots, k_{r} \in \mathbf{N}_{0} \\
\left(\ell_{2} k_{2}+\cdots+\ell_{r} k_{r}=\ell_{1} n-k_{1}\right)
\end{array}} \mathcal{U}_{k_{1}, \ell_{1}, \cdots, \ell_{r}}^{\left(\alpha_{1}, \cdots, \alpha_{r}\right)}\left(x_{1}, \cdots, x_{r}\right) \\
\cdot \frac{\left\langle-\alpha_{2} x_{2}\right\rangle^{k_{2}}}{k_{2} !} \cdots \frac{\left\langle-\alpha_{r} x_{r}\right\rangle^{k_{r}}}{k_{r} !},
\end{gathered}
$$


which, in the special case when $\ell_{j}=1(j=1, \cdots, r)$, the monoumbral expansion given by (3.6). Moreover, if we simply assume that $\ell_{r}=1$, we can easily rewrite the monoumbral expansion (3.12) as follows:

$$
\begin{gathered}
x_{1}^{n}=\frac{n !}{\left(\alpha_{1}\right)_{n}} \sum_{k_{1}=0}^{\ell_{1} n} \sum_{k_{2}=0}^{\left[\frac{\ell_{1} n-k_{1}}{\ell_{2}}\right]} \ldots \sum_{k_{r-1}=0}^{\left[\frac{\ell_{1} n-k_{1}-\ell_{2} k_{2}-\cdots-\ell_{r-2} k_{r-2}}{\ell_{r-1}}\right]} \\
\cdot \mathcal{U}_{k_{1} ; \ell_{1}, \cdots, \ell_{r-1}, 1}^{\left(\alpha_{1}, \alpha_{r}\right)}\left(x_{1}, \cdots, x_{r}\right) \\
\cdot \frac{\left\langle-\alpha_{2} x_{2}\right\rangle^{\ell_{1} n-k_{1}-\ell_{2} k_{2}-\cdots-\ell_{r-1} k_{r-1}}}{\left(\ell_{1} n-k_{1}-\ell_{2} k_{2}-\cdots-\ell_{r-1} k_{r-1}\right) !} \frac{\left\langle-\alpha_{3} x_{3}\right\rangle^{k_{2}}}{k_{2} !} \cdots \frac{\left\langle-\alpha_{r} x_{r}\right\rangle^{k_{r-1}}}{k_{r-1} !}
\end{gathered}
$$

or, equivalently,

$$
\begin{gathered}
x_{1}^{n}=\frac{n !}{\left(\alpha_{1}\right)_{n}} \sum_{k_{1}=0}^{\ell_{1} n} \sum_{k_{2}=0}^{\left[\frac{\ell_{1} n-k_{1}}{\ell_{2}}\right]} \cdots \sum_{k_{r-1}=0}^{\left[\frac{\ell_{1} n-k_{1}-\ell_{2} k_{2}-\cdots-\ell_{r-2} k_{r-2}}{\ell_{r-1}}\right]} \\
\cdot \mathcal{U}_{k_{1} ; \ell_{1}, \cdots, \ell_{r-1}, 1}^{\left(\alpha_{1}, \cdots, \alpha_{r}\right)}\left(x_{1}, \cdots, x_{r}\right) \\
\frac{k_{1} !\left(\ell_{2} k_{2}\right) ! \cdots\left(\ell_{r-1} k_{r-1}\right) !}{k_{2} ! \cdots k_{r-1} !}\left(\begin{array}{c}
\ell_{1} n \\
k_{1}
\end{array}\right)\left(\begin{array}{c}
\ell_{1} n-k_{1} \\
\ell_{2} k_{2}
\end{array}\right) \\
\ldots\left(\begin{array}{c}
\ell_{1} n-k_{1}-\ell_{2} k_{2}-\cdots-\ell_{r-2} k_{r-2} \\
\ell_{r-1} k_{r-1}
\end{array}\right) \\
\cdot\left\langle-\alpha_{2} x_{2}\right\rangle^{\ell_{1} n-k_{1}-\ell_{2} k_{2}-\cdots-\ell_{r-1} k_{r-1}}\left\langle-\alpha_{3} x_{3}\right\rangle^{k_{2} \cdots\left\langle-\alpha_{r} x_{r}\right\rangle^{k_{r-1}}}
\end{gathered}
$$

in terms of the notations and conventions given by (3.7) and (3.8). Indeed, when we further set $\ell_{j}=1(j=1, \cdots, r-1)$, this last result (3.14) would correspond precisely to the monoumbral expansion (3.6).

Finally, upon setting $\ell_{j}=j \quad(j=1, \cdots, r)$ in our general result (3.12), we are led at once to the following monoumbral expansion for the multivariable Lagrange-Hermite polynomials $h_{n}^{\left(\alpha_{1}, \cdots, \alpha_{r}\right)}\left(x_{1}, \cdots, x_{r}\right)$ in (1.6), which were studied by (for example) Altın and Erkuş [1] :

$$
x_{1}^{n}=\frac{n !}{\left(\alpha_{1}\right)_{n}} \sum_{k_{1}=0}^{n} \sum_{\substack{k_{2}, \cdots, k_{r} \in \mathbf{N}_{0} \\\left(2 k_{2}+\cdots+r k_{r}=n-k_{1}\right)}} h_{k_{1}}^{\left(\alpha_{1}, \cdots, \alpha_{r}\right)}\left(x_{1}, \cdots, x_{r}\right)
$$




$$
\cdot \frac{\left\langle-\alpha_{2} x_{2}\right\rangle^{k_{2}}}{k_{2} !} \cdots \frac{\left\langle-\alpha_{r} x_{r}\right\rangle^{k_{r}}}{k_{r} !}
$$

\section{Concluding Remarks and Observations}

In this work, we have investigated the umbral calculus presentations of the Chan-Chyan-Srivastava polynomials $g_{n}^{\left(\alpha_{1}, \cdots, \alpha_{r}\right)}\left(x_{1}, \cdots, x_{r}\right)$ generated by (1.3) and also of the substantially more general Erkuş-Srivastava polynomials $\mathcal{U}_{n ; \ell_{1}, \cdots, \ell_{r}}^{\left(\alpha_{1}, \cdots, \alpha_{r}\right)}\left(x_{1}, \cdots, x_{r}\right)$ generated by (1.7). One of our main monoumbral expansions asserted by (3.12) has been shown to yield the corresponding monoumbral expansion (3.15) for the multivariable Lagrange-Hermite polynomials $h_{n}^{\left(\alpha_{1}, \cdots, \alpha_{r}\right)}\left(x_{1}, \cdots, x_{r}\right)$ in (1.6), which were studied by (for example) Altin and Erkuş [1].

We need hardly emphasize upon the fact that the notion and formalism of monoumbrality can be extended to a large family of polynomials as well as of functions and that the underlying principle of monoumbrality would provide a powerful tool for simplifying calculations.

We conclude our present investigation by remarking further that, in the special case when $\ell_{1}=1$, we find from (1.8) that

$$
\begin{aligned}
& \mathcal{U}_{n ; 1, \ell_{2}, \cdots, \ell_{r}}^{\left(\alpha_{1}, \cdots, \alpha_{r}\right)}\left(x_{1}, \cdots, x_{r}\right)=\sum_{\substack{k_{1}, \cdots, k_{r} \in \mathbf{N}_{0} \\
\left(k_{1}+\ell_{2} k_{2}+\cdots+\ell_{r} k_{r}=n\right)}} \frac{\left\langle\alpha_{1} x_{1}\right\rangle^{k_{1}}}{k_{1} !} \cdots \frac{\left\langle\alpha_{r} x_{r}\right\rangle^{k_{r}}}{k_{r} !} \\
& =\sum_{k_{2}, \ldots, k_{r}=0} \stackrel{\left\langle\alpha_{1} x_{1}\right\rangle^{n-\ell_{2} k_{2}-\cdots-\ell_{r} k_{r}}}{\left(n-\ell_{2} k_{2}-\cdots-\ell_{r} k_{r}\right) !} \frac{\left\langle\alpha_{2} x_{2}\right\rangle^{k_{2}}}{k_{2} !} \cdots \frac{\left\langle\alpha_{r} x_{r}\right\rangle^{k_{r}}}{k_{r} !} \\
& =\sum_{k_{1}=0}^{\left[\frac{n}{\ell_{2}}\right]} \sum_{k_{2}=0}^{\left[\frac{n-\ell_{2} k_{1}}{\ell_{3}}\right]} \cdots \sum_{k_{r-1}=0}^{\left[\frac{n-\ell_{2} k_{1}-\cdots-\ell_{r-1} k_{r-2}}{\ell_{r}}\right]} \\
& \cdot \frac{\left\langle\alpha_{1} x_{1}\right\rangle^{n-\ell_{2} k_{1}-\cdots-\ell_{r} k_{r-1}}}{\left(n-\ell_{2} k_{1}-\cdots-\ell_{r} k_{r-1}\right) !} \frac{\left\langle\alpha_{2} x_{2}\right\rangle^{k_{1}}}{k_{1} !} \cdots \frac{\left\langle\alpha_{r} x_{r}\right\rangle^{k_{r-1}}}{k_{r-1} !} \text {, }
\end{aligned}
$$

which readily yields the following generalization of the operational formula (2.6):

$$
\left(\hat{X}_{1} \hat{P}_{x_{1}}+\ell_{2} \hat{X}_{2} \hat{P}_{x_{2}}+\cdots+\ell_{r} \hat{X}_{r} \hat{P}_{x_{r}}\right)\left\{\mathcal{U}_{n ; 1, \ell_{2}, \cdots, \ell_{r}}^{\left(\alpha_{1}, \cdots, \alpha_{r}\right)}\left(x_{1}, \cdots, x_{r}\right)\right\}
$$




$$
=n \mathcal{U}_{n ; 1, \ell_{2}, \cdots, \ell_{r}}^{\left(\alpha_{1}, \cdots, \alpha_{r}\right)}\left(x_{1}, \cdots, x_{r}\right)
$$

In particular, if we set $\ell_{j}=j(j=2, \cdots, r)$ in this last result (4.2), we immediately obtain

$$
\begin{gathered}
\left(\hat{X}_{1} \hat{P}_{x_{1}}+2 \hat{X}_{2} \hat{P}_{x_{2}}+\cdots+r \hat{X}_{r} \hat{P}_{x_{r}}\right)\left\{h_{n}^{\left(\alpha_{1}, \cdots, \alpha_{r}\right)}\left(x_{1}, \cdots, x_{r}\right)\right\} \\
=n h_{n}^{\left(\alpha_{1}, \cdots, \alpha_{r}\right)}\left(x_{1}, \cdots, x_{r}\right)
\end{gathered}
$$

for the multivariable Lagrange-Hermite polynomials $h_{n}^{\left(\alpha_{1}, \cdots, \alpha_{r}\right)}\left(x_{1}, \cdots, x_{r}\right)$ in (1.6), which were studied by (for example) Altın and Erkuş [1]. Indeed, in its further special case when

$$
r=2, \quad \alpha_{1}=\alpha, \quad \alpha_{2}=\beta, \quad x_{1}=x \quad \text { and } \quad x_{2}=y,
$$

the operational formula (4.3) would provide the corrected version of another result stated by Dattoli et al. [6, p. 184, Eq. (22)].

\section{References}

[1] A. Altın and E. Erkuş, On a multivariable extension of the LagrangeHermite polynomials, Integral Transforms Spec. Funct. 17, pp. 239244, (2006).

[2] L. C. Andrews, Special Functions for Engineers and Applied Mathematicians, Macmillan Company, New York, (1985).

[3] W.-C. C. Chan, C.-J. Chyan and H. M. Srivastava, The Lagrange polynomials in several variables, Integral Transforms Spec. Funct. 12, pp. 139-148, (2001).

[4] K.-Y. Chen, S.-J. Liu and H. M. Srivastava, Some new results for the Lagrange polynomials in several variables, ANZIAM J. 49, pp. 243258, (2007).

[5] G. Dattoil, P. E. Ricci and C. Cesarano, Monumbral polynomials and the associated formalism, Integral Transforms Spec. Funct. 13, pp. 155-162, (2002). 
[6] G. Dattoil, P. E. Ricci and C. Cesarano, The Lagrange polynomials, the associated generalizations, and the umbral calculus, Integral Transforms Spec. Funct. 14, pp. 181-186, (2003).

[7] G. Dattoil, P. E. Ricci and C. Cesarano, Beyond the monomiality: The monumbrality principle, J. Comput. Anal. Appl. 6, pp. 77-83, (2004).

[8] A. Erdélyi, W. Magnus, F. Oberhettinger and F. G. Tricomi, Higher Transcendental Functions, Vol. III, McGraw-Hill Book Company, New York, Toronto and London, (1955).

[9] E. Erkus and H. M. Srivastava, A unified presentation of some families of multivariable polynomials, Integral Transforms Spec. Funct. 17, pp. 267-273, (2006).

[10] M. A. Khan and A. K. Shukla, On Lagrange's polynomials of three variables, Proyecciones J. Math. 17, pp. 227-235, (1998).

[11] S.-J. Liu, Bilateral generating functions for the Lagrange polynomials and the Lauricella functions, Integral Transforms Spec. Funct. 20, pp. 519-527, (2009).

[12] S.-J. Liu, C.-J. Chyan, H.-C. Lu and H. M. Srivastava, Bilateral generating functions for the Chan-Chyan-Srivastava polynomials and the generalized Lauricella functions, Integral Transforms Spec. Funct. 23, pp. 539-549, (2012).

[13] S.-J. Liu, S.-D. Lin, H. M. Srivastava and M.-M. Wong, Bilateral generating functions for the Erkuş-Srivastava polynomials and the generalized Lauricella functions, Appl. Math. Comput. 218, pp. 7685-7693, (2012).

[14] S. Roman, The Umbral Calculus, Academic Press, New York and London, (1984).

[15] H. M. Srivastava and H. L. Manocha, A Treatise on Generating Functions, Halsted Press (Ellis Horwood Limited, Chichester), John Wiley and Sons, New York, Chichester, Brisbane and Toronto, (1984). 
H. M. Srivastava

Department of Mathematics and Statistics, University of Victoria, Victoria, British Columbia V8W 3R4, Canada

e-mail : harimsri@math.uvic.ca

K. S. Nisar

Department of Mathematics, College of Engineering, Salman Bin Abdul-Aziz University, Wadi Dawaser,

Kingdom of Saudi Arabia

e-mail : ksnisar1@gmail.com

and

\section{Mumtaz Ahmad Khan}

Department of Applied Mathematics, Faculty of Engineering and Technology, Aligarh Muslim University, Aligarh 202002, Uttar Pradesh, India

e-mail : mumtaz_ahmad_khan_2008@yahoo.com 